\title{
Determination of Array Gain of Single Hop to Achieve the Performance of a 2-Hop Wireless Link
}

\author{
Abu Sayed Md. Mostafizur Rahaman ${ }^{1}$, Jesmin Akhter ${ }^{2}$, Md. Rafsan Jani ${ }^{1}$, Md. Imdadul Islam ${ }^{1}$ \\ ${ }^{1}$ Department of Computer Science and Engineering, Jahangirnagar University, Savar, Dhaka, Bangladesh \\ ${ }^{2}$ Institute of Information Technology, Jahangirnagar University, Savar, Dhaka, Bangladesh \\ Email:imdad@juniv.edu
}

How to cite this paper: Rahaman, A.S.Md.M., Akhter, J., Jani, Md.R. and Islam, Md.I. (2018) Determination of Array Gain of Single Hop to Achieve the Performance of a 2-Hop Wireless Link. Journal of Computer and Communications, 6, 84-98. https://doi.org/10.4236/jcc.2018.67008

Received: July 1, 2018

Accepted: July 28, 2018

Published: July 31, 2018

Copyright $\odot 2018$ by authors and Scientific Research Publishing Inc. This work is licensed under the Creative Commons Attribution International License (CC BY 4.0).

http://creativecommons.org/licenses/by/4.0/

\begin{abstract}
In adaptive beamforming system adaptive algorithm of digital filter is applied to update the weighting vector of the antenna elements to get antenna gain along the desired direction and attenuation along the jammer. The objective of the paper is to evaluate the threshold gain of the adaptive beam former along the line of sight (LOS) between the transmitter and the receiver (including jammer suppression) to make the single hop link comparable with 2-hop link. The single hop and 2-hop communication systems are compared in context of symbol error rate (SER) under fading condition theoretically and verified by simulation. Finally we evaluate the numerical value of threshold gain of adaptive beamformer of two antenna elements under Rayleigh and Nakagami- $m$ fading conditions.
\end{abstract}

\section{Keywords}

Array Antenna System, BER, Threshold Array Gain, Side Lobe Canceller, Path Loss

\section{Introduction}

To combat the fading affect of long wireless link, 2-hop model is widely used where a relay is placed between transmitter and receiver. The concept is applicable to enhance the performance of point to point link. The combined SNR of two links becomes approximately parallel combination of two SNRs like equivalent parallel resistor of electrical circuit. Therefore the combined SNR is less than the individual SNR (between transmitter and repeater or repeater and receiver) but the SNR between the transmitter and receiver (without the repeater station) is 
much less than the combined SNR of 2-hop wireless link. This phenomenon attracts the researcher to apply the concept of multi-hop in wireless networks to reduce SER and enhance throughput. The performance analysis of two-hop wireless link in context of BER under fading is discussed in [1] [2] [3] [4]. Usually in rural or suburban area the gain of the relay is kept constant called amplify-and-forward (AF) relaying. In a dense urban area the wireless link is found as time selective channel i.e. SNR of receiver varies with time hence the gain of the relay has to be adjusted with feedback signal like adaptive algorithm of digital filter. The concept of AF and DF relay is found in [5] [6] [7] where the idea of channel state information is included. In [8], authors analyzed performance of AF relay network where relay is affected by interferences under assumption that there is no obstacle between interferences and relay i.e. LOS interferences. The paper derived closed form solution of outage probability with the idea of [9] [10]. To reduce BER of 3G mobile cellular destined for high speed communication needs to overcome small scale fading (Rayleigh fading). In this context selection of best relay is discussed in [11] with MIMO (multiple input multiple output). Actually MIMO is used in wireless link to take the advantage of space diversity at the expense of system cost. Different combination of MIMO (both the hop is MIMO, MISO in first hop and SIMO in second hop, SIMO in first hop and MISO in second hop etc.) is found in recent literature for 2-hop wireless link [11] [12] [13].

The main objective of AAS is to combine the radiation pattern of individual antenna element (with proper weighting factor and phase shift) to produce a combined beam of desired directivity and gain. The detail analysis of AAS is found in [14] [15] [16]. To make the system dynamic, adaptive beam forming algorithm is used to select the appropriate weighting factor of antenna elements. Several applications of AAS for example: Beam Steering and Switching, Conventional Beam Forming, Diversity Combining, Array on Satellites, Dynamic beams, Reduction in Delay Spread and Multipath Fading, Reduction in Co-channel Interference etc. are discussed in [14]. The same author discussed detail about mathematical model and adaptive algorithms of conventional beam former, null-steering beam former, optimal beam forming, broad-band beam forming, frequency-domain beam forming, SMI algorithm, LMS algorithm, RLS algorithm, MVDR estimator etc in [15]. In this paper we make a comparison of 2-hop wireless link under single antenna system with the case of direct wireless link (without relay) of array antenna system (AAS) to reduce complexity of communication system. Two most important features of 5G mobile communication are: "Ultra-Dense Networks (UDN)" and "massive MIMO" hence multi-hop wireless link will make the system very complex. The objective of the paper is to acquire the capacity of single hop link equivalent to dual hop link. The adaptive beamformer can only help us to achieve the goal.

The paper is organized like: Section 2 provides the theoretical analysis of dual-hop wireless link in determination of overall SNR, Section 3 deals with 
concept of AAS, overall SINR (signal to interference and noise ratio) at receiving end and algorithms of adaptive beamforming; Section 4 provides the results based on theoretical analysis of Section 3; and Section 5 concludes the entire analysis.

\section{Dual-Hop Wireless Link}

First of all we consider the 2-hop wireless link of Figure 1 where the SNR between transmitter and relay is $\Gamma_{S R}$ and that of relay to destination is $\Gamma_{R D}$.

The received signal vector at the relay is expressed as:

$$
\boldsymbol{y}_{R}=\sqrt{E} \boldsymbol{H}_{S R} \boldsymbol{x}+\boldsymbol{n}_{S R},
$$

where $E$ is the transmitted power, $\boldsymbol{H}_{S R}$ is the channel matrix from the source to relay, $\boldsymbol{x}$ is the signal vector of source, and $\boldsymbol{n}_{S R}$ is the noise vector of source to relay link. Above expression is applicable for MIMO system but for single antenna system instead of signal vector we consider individual symbol (for example symbols of 16-QAM) of transmitter and instead of channel matrix we consider simply channel gain may be complex number. When the signal is detected at relay, it is processed and transmitted with some delay expressed as: $\boldsymbol{y}_{R D}^{\prime}$ hence the received signal vector at the destination is,

$$
\boldsymbol{y}_{D}=\sqrt{E} \boldsymbol{H}_{R D} \boldsymbol{y}_{R D}^{\prime}+\boldsymbol{n}_{R D}
$$

The noise vector on both the links are uncorrelated and possesses the properties: $\mathrm{E}\left(n_{S R} n_{S R}^{\dagger}\right)=I_{N_{R}} N_{0}$ and $\mathrm{E}\left(n_{R D} n_{R D}^{\dagger}\right)=I_{N_{D}} N_{0}$, where $N_{0}$ is the variance of the additive white Gaussian noise (AWGN), $I_{N_{R}}$ is the identity matrix with $N_{R}$ antennas at the relay station and $N_{D}$ is the number of antennas at the destination.

If the SNR of the links are: $\Gamma_{S R}=E_{S}\left\|H_{S R}\right\|^{2} / N_{0}$ and $\Gamma_{R D}=\bar{E}_{R}\left\|H_{R D}\right\|^{2} / N_{0}$, then the overall SNR will be [4] [11]:

$$
\Gamma_{S D}=\frac{\Gamma_{S R} \Gamma_{R D}}{\Gamma_{S R}+\Gamma_{R D}+1}
$$

This overall SNR will be compared with overall SNR of AAS with adaptive beamformer under single hop of same path length.

For single antenna case Equations (1) and (2) is written conventionally like:

$$
\begin{gathered}
y_{R}=\sqrt{P} h_{S R} x+n_{S R} \\
y_{D}=\sqrt{P} h_{R D} y_{R}^{\prime}+n_{R D}
\end{gathered}
$$

where each variable indicates a real or complex number instead of vector.

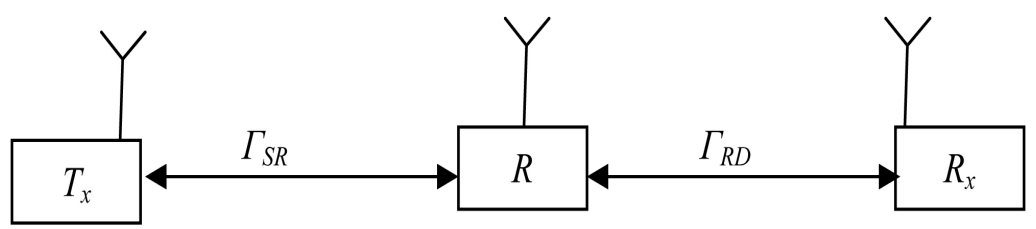

Figure 1. Dual-hop wireless link. 
Once combined SNR $\gamma_{c}=\Gamma_{S D}$ is evaluated then the SER under awgn is expressed as [8],

$$
P_{s}\left(\gamma_{c}\right)=a Q\left(\sqrt{2 b \gamma_{c}}\right)
$$

where $\gamma_{c}$ is a r.v. and the integers $a$ and $b$ depend on type of modulation scheme. Above analysis under fading channel is expressed as,

$$
P_{s}\left(\gamma_{c_{-} a v}\right)=\int_{0}^{\infty} P_{s}\left(\gamma_{c}\right) f_{\Gamma_{c}}\left(\gamma_{c}, \gamma_{c_{-} a v}\right) \mathrm{d} \gamma_{c}
$$

where $f_{\Gamma_{c}}\left(\gamma_{c}, \gamma_{c_{-} a v}\right)$ is the pdf of r.v. of small scale fading channel and $\gamma_{c_{-} a v}$ is the average value of the r.v. In this paper we consider only Nakagami- $m$ fading channel. For example for Nakagami- $m$ fading case the pdf becomes,

$$
f_{\Gamma_{c}}\left(\gamma_{c}, \gamma_{c_{-} a v}\right)=\frac{m^{m} \gamma_{c}^{m-1}}{\gamma_{c_{-} a v}^{m} \Gamma(m)} \mathrm{e}^{-m \gamma_{c}^{2} / \gamma_{c_{-} a v}}
$$

We can use Equations (7) and (8) to evaluate SER under Nakagami- $m$ fading case.

\section{Adaptive Beamforming}

In array antenna system the number of antenna elements is a vital factor to achieve a wide variation of directivity and gain visualized from Figure 2; where a linear array is considered with spacing of antenna elements of half wavelength i.e. $\lambda / 2$.

Extraction of the original signal from a noisy environment is achieved by Finite Impulse Response (FIR) or Infinite Impulse Response (IIR) filter but their coefficients remain fixed during entire operation. In adaptive filter, adaptive

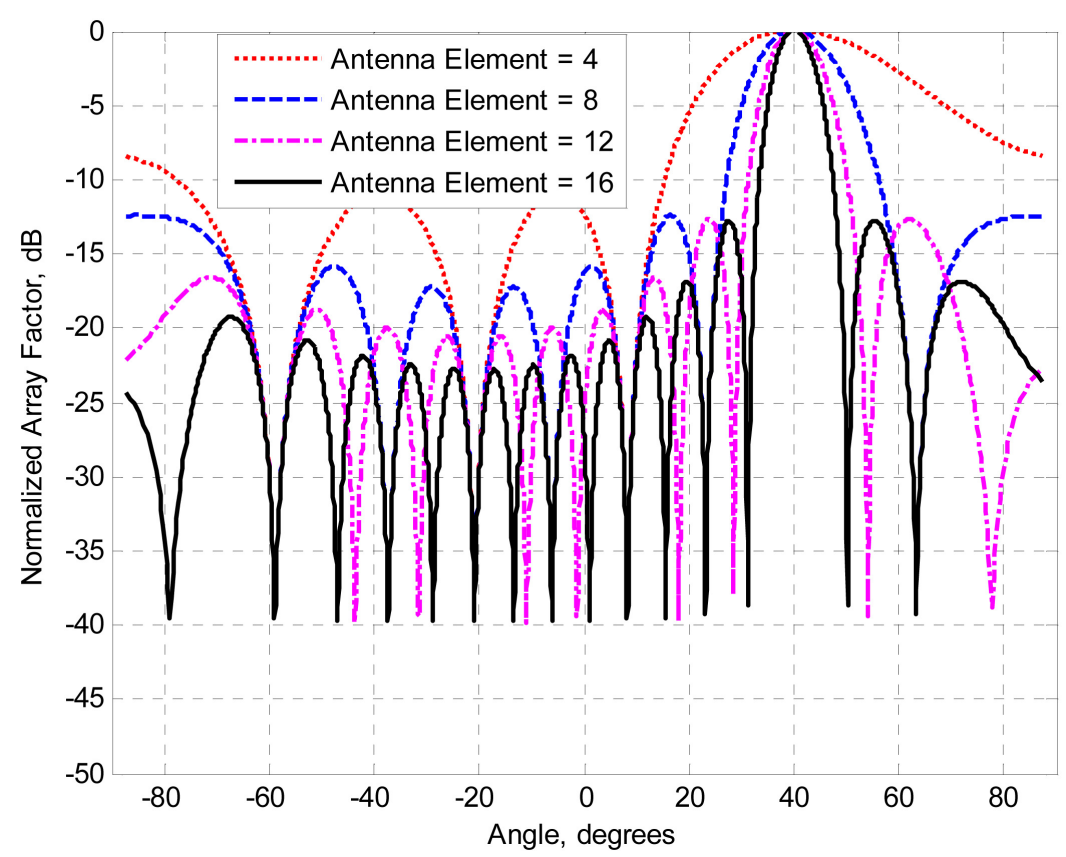

Figure 2. Radiation pattern of linear array taking spacing of array elements $\lambda / 2$. 
algorithms are used to adjust the coefficients of the digital filter so that the difference between the noisy input signal, $X_{k}$ and the desired signal, $d$ called the error signal $\varepsilon$ attains at minimum value. The common algorithms found widespread applications are the least mean square (LMS), the recursive least squares (RLS), and the Kalman filter algorithms. In terms of computational complexity and storage requirements, the LMS algorithm is the most efficient but the Kalman filter is suitable for immunity of any type of noise at the expense of process time. To enhance the gain of the antenna array in the direction of desired signal and to provide attenuation in the direction of interference or multiple jammer we use adaptive algorithm of IIR and FIR filter. An adaptive filter consists of FIR/IIR and adaptive algorithm to adjust the weighting factor of each branch of signal discussed in [17] [18], such concept in AAS is called adaptive beamforming. The following two sub-sections will provide the basic concept of adaptive beamforming technique where the concept is available in [19] [20].

Let us now provide the way of enhancement of gain in desired direction and elimination of jammers using the concept of adaptive beamforming. We will then determine the threshold gain of the beamformer under a single hop wireless link to be comparable with case of 2-hop link in context of SER. In adaptive beam former two types of omni antennas are used 1) primary antenna 2) reference antenna. Both types of antennas receive signal and interference/jammer. Each reference antenna element is associated with adaptive filter and its weight is adjusted in such a way that it can receive only jammer where the primary antenna provides the sum of signal and jammer. Now the jammer part of the primary antenna is correlated with the output of reference antenna hence the error between primary and reference antenna provides the required signal component. The weighting factors of reference antenna elements are updated using adaptive algorithms of [21].

To match with multiple jammers of different direction at the same time, it is necessary to have more than one reference omni as explained in [22] [23]. Figure 3 shows the arrangement of such antenna system considering two spatially separated reference omnis.

Radiation pattern of two reference adaptive beamforming is shown in Figure 4 taking the weighting factors as: $w_{11}=-0.48, w_{12}=-0.871, w_{21}=1.7$ and $w_{22}=1$ based on [24]. Here the null forms along $30^{\circ}, 150^{\circ}, 60^{\circ}$ and $120^{\circ}$; where the main beam is directed along $270^{\circ}$.

$$
\text { The overall SINR at receiving end is, } \Gamma_{0}=\frac{P_{r} \cdot G_{d}}{\sum_{j=1}^{k} I_{j} \cdot A_{j}+N_{0}} \approx \Gamma \cdot G
$$

where $P_{r}$ is the received signal power along desired direction, $G_{d}$ is gain of the AAS along the link between $T_{x}$ and $R_{x}, A_{j}$ is the gain along the $j$ th jammer, $I_{j}$ is the interference/jammer along $j$ th direction and $N_{0}$ is the awgn noise. Overall SINR is approximated as $\Gamma \cdot G$; where $\Gamma$ is the SNR of conventional single antenna system and $G$ is the ratio of $G_{d}$ and $A_{j}$. 


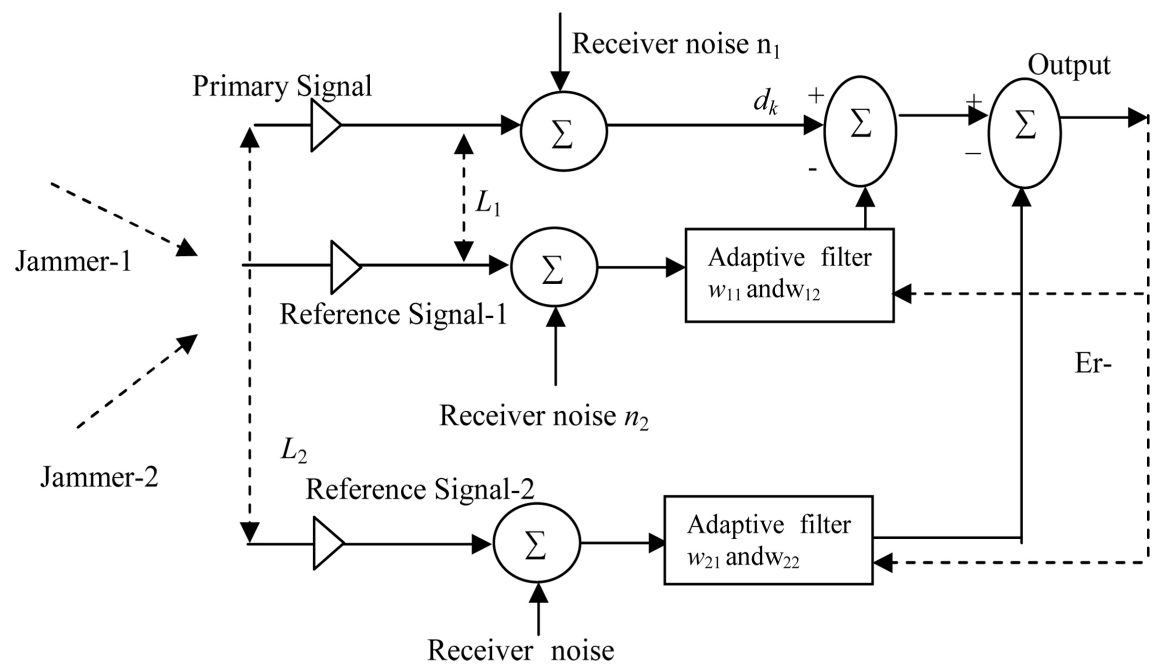

Figure 3. Side lobe canceller with two incident signals.

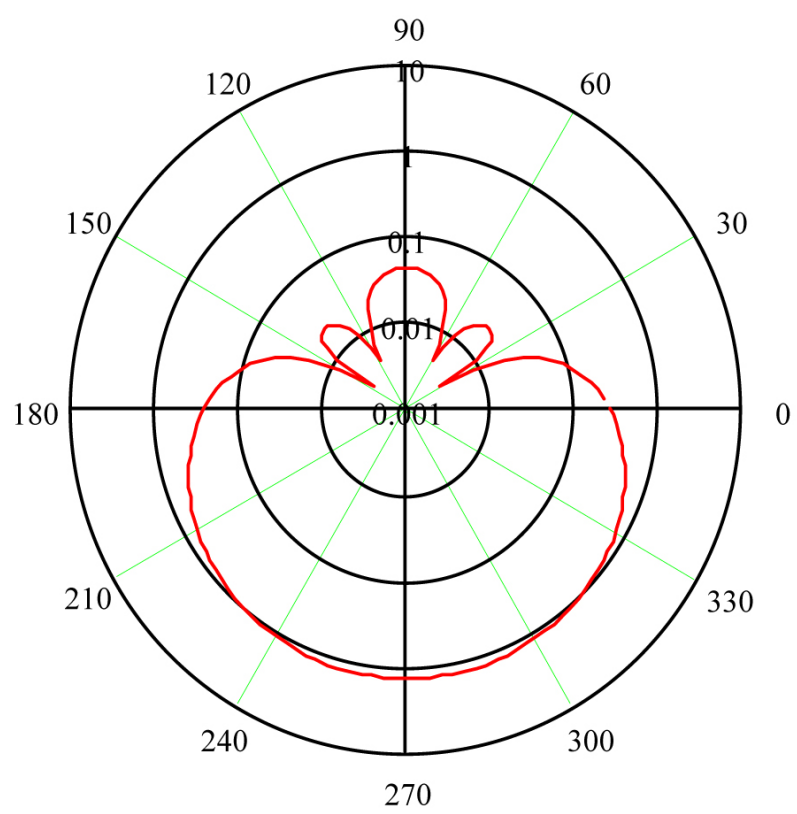

Figure 4. Radiation pattern of two reference antenna system.

In free space path loss model the received signal at a distance $d$ is expressed as: $P_{r}=K \cdot P_{t} \cdot d^{-\alpha}$; where $\alpha$ is path-loss exponent. Therefore if the distance is increased to twice of the previous one then the received signal or SNR is decreased by a factor of $2^{-\alpha}$. The overall SNR of 2-hop wireless link (distance of each is $d$ ) is approximately half of SNR of single hop link of distance $d$. If the enhancement of overall gain $G$ of the array antenna system is greater than $2^{-\alpha-1}$ then the proposed scheme of the thesis brings the desired result will be verified in next section. Such concept is available in [25] [26] in a different way. In real life the situation is the analysis becomes more complicated due to large scale fading (modeled by Okumura-Hata/Walfish-Ikegami/Lee) and small scale fading (modeled by FIR filter with appropriated fading parameters). 


\subsection{Algorithm-1}

In this algorithm we use the concept of Wiener filter theory in minimization of mean square error of cost function which actually represents the difference between output of the adaptive filter and desired output. For input signal $u(n)$ and $k$ th weighting factor $W_{k}$ the output signal from a linear combiner is given as,

$$
y(n)=\sum_{k=0}^{M-1} W_{k}^{*} u(n-k)
$$

Output power,

$$
P=|y(n)|^{2}=\sum_{k=0}^{M-1} \sum_{i=0}^{M-1} W_{k}^{*} W_{i} E[u(n-k) u(n-i)]=\sum_{k=0}^{M-1} \sum_{i=0}^{M-1} W_{k}^{*} W_{i} r(i-k)
$$

where $E[u(n-k) u(n-i)]=r(i-k)$ is the autocorrelation function.

If the beamformer is subjected to a linear constraint, $\sum_{k=0}^{M-1} W_{k}^{*} \mathrm{e}^{-j \theta_{0} k}=g$; then the real valued cost function becomes,

$$
J=\sum_{k=0}^{M-1} \sum_{i=0}^{M-1} W_{k}^{*} W_{i} r(i-k)+\operatorname{Re}\left[\lambda^{*}\left(\sum_{k=0}^{M-1} W_{k}^{*} \mathrm{e}^{-j \theta_{0} k}-g\right)\right]
$$

where $g$ is the complex-valued gain, $\theta_{0}$ desired electrical angle of arrival.

Taking gradient of $J$ and putting the result equal to zero like Wiener filter theorem, we get the optimum weighting vector like,

$$
W_{0}=\frac{g^{*} R^{-1} S\left(\theta_{0}\right)}{S^{H}\left(\theta_{0}\right) R^{-1} S\left(\theta_{0}\right)}
$$

where $S\left(\theta_{0}\right)=\left[\begin{array}{lllll}1 & \mathrm{e}^{-j \theta_{0}} & \mathrm{e}^{-j 2 \theta_{0}} & \cdots & \mathrm{e}^{-j(M-1) \theta_{0}}\end{array}\right]$ is known as steering vector and its details are found in [27] [28] [29].

Considering uniform linear array of $N=6$ antenna elements the variation of normalized power $(\mathrm{dB})$ against the azimuth angle is shown in Figure 3 taking the AOA of: azimuth $60^{\circ}$, elevation $15^{\circ}$ and carrier frequency of $f_{c}=1 \mathrm{GHz}$. The response is found different after inclusion of steering vector, visualized the Figure 5.

The steering vector corresponding to Figure 5 is,

$$
\begin{aligned}
& \boldsymbol{S}=\left[\begin{array}{lll}
-0.9971-0.0760 i & 0.8350-0.5503 i & -0.3234+0.9462 i
\end{array}\right. \\
& -0.3234-0.9462 i \quad 0.8350+0.5503 i \quad-0.9971+0.0760 i]^{\mathrm{T}}
\end{aligned}
$$

\subsection{Algorithm-2}

This algorithm is an extension of previous one where the desired and undesired signals are analyzed separately. A widely used method of adaptive beamforing is LCMV (linearly constraint minimum variance) algorithm where the weighting vector of adaptive filter is divided into two portion $W_{q}$ and $W_{a}$. Here the first one is dependent on desired signal i.e. signal along the desired AOA (Angle of Arrival) and the second one is related to sidelobe and un-correlated with the desired signal. A constraint matrix $C_{a}$ correspond to a band rejection filter is used to segregate the sidelobe from the desired signal shown in Figure 6. Finally 

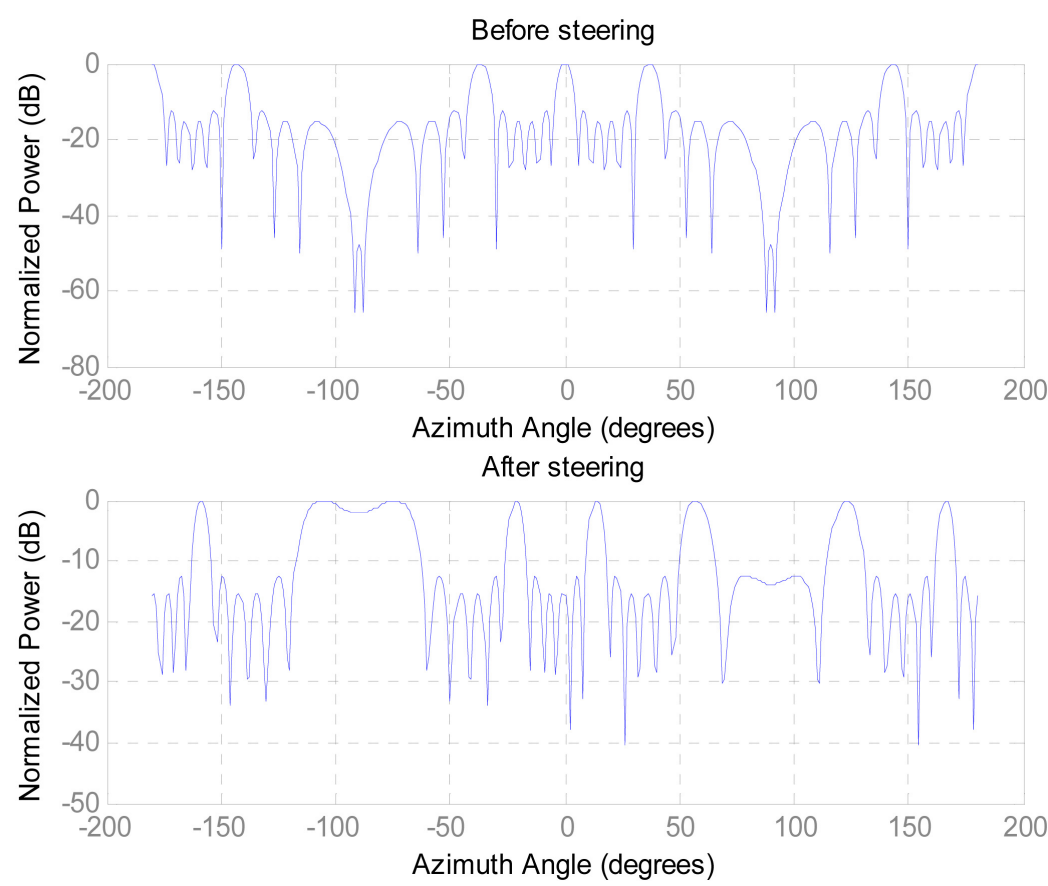

Figure 5. The profile of normalized power against azimuth angle.

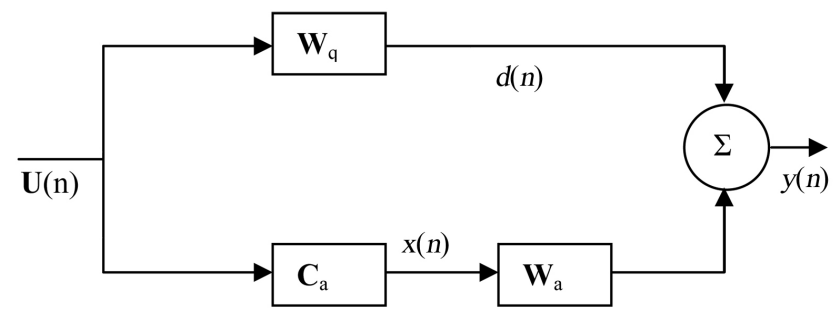

Figure 6. Adaptive beamformer with LCMV.

Winner filter theorem is applied to in getting weighting vector for minimum output $y(n)$.

Let us introduce multiple linear constraints by the constraint matrix $C$ and gain vector $\boldsymbol{g}$ like,

$$
\boldsymbol{C}^{H} \boldsymbol{W}=\boldsymbol{g}
$$

where the overall weight vector is, $\boldsymbol{W}=\boldsymbol{W}_{q}-\boldsymbol{C}_{a} \boldsymbol{W}_{a}$

The desired signal of the beamformer,

$d(n)=\boldsymbol{W}_{q}^{H} \cdot \boldsymbol{U}(n) ;$ where $\boldsymbol{W}_{q}=\boldsymbol{C}\left(\boldsymbol{C}^{H} \boldsymbol{C}\right)^{-1} \boldsymbol{g}$

If $C_{a}$ is a stop band FIR filter with zero response in desired direction $\theta_{0}$ then the signal with sidelobe is $\boldsymbol{C}_{a}^{H} \boldsymbol{U}(n)=x(n)$ and the output signal is $y(n)=d(n)-\boldsymbol{W}_{q}^{H} x(n)$.

Applying Winner filter theorem,

$$
\boldsymbol{W}_{a}=\left(\boldsymbol{C}_{a}^{H} \boldsymbol{R} \boldsymbol{C}_{a}\right)^{-1} \boldsymbol{C}_{a}^{H} \boldsymbol{R} \boldsymbol{W}_{q}
$$

where $R$ is the autocorrelation matrix of input vector. 
Taking the following parameters under LCMV: SNR $=50 \mathrm{~dB}$, carrier frequency $f_{c}=1 \mathrm{GHz}$, the number of antenna elements $N=10$, antenna spacing $\lambda / 2$ and elevation angles $0^{\circ}$ we get the following response (using simulation of LCMV in Matlab 13) for different AOA and directions of jammer (azimuth angle) shown in Figure 7. Such concept is available for both linear and circular array in [30].

The weighting vectors for above 4 cases are evaluated as:

$$
\begin{aligned}
& W_{1}=\left[\begin{array}{lllll}
-0.0489-0.1267 i & 0.0443+0.1161 i & -0.0540-0.0985 i & 0.0322+0.0688 i
\end{array}\right. \\
& \begin{array}{llll}
-0.0176-0.1005 i & -0.0264+0.0665 i & 0.0128-0.0669 i & -0.0613+0.1043 i
\end{array} \\
& 0.0407-0.1328 i-0.0558+0.1188 i]^{\mathrm{T}} \\
& W_{2}=\left[\begin{array}{lll}
0.0680-0.0776 i & 0.0364+0.0809 i-0.0568+0.0648 i-0.0782-0.0738 i
\end{array}\right. \\
& 0.0767-0.0872 i \quad 0.0847+0.0737 i-0.0767+0.0672 i-0.0731-0.0556 i \\
& 0.0619-0.0593 i \quad 0.0786+0.0830 i]^{\mathrm{T}} \\
& W_{3}=\left[\begin{array}{llll}
0.0452-0.0686 i-0.0665+0.0640 i & 0.0487-0.0840 i-0.0318+0.0945 i
\end{array}\right. \\
& 0.0142-0.1566 i-0.0035+0.1070 i-0.0446-0.0866 i \quad 0.0445+0.0989 i \\
& -0.0491-0.0701 i \quad 0.0633+0.0493 i]^{\mathrm{T}} \\
& W_{4}=\left[\begin{array}{llll}
-0.0009-0.0962 i-0.0208+0.0956 i & -0.0021-0.1051 i & 0.0014+0.0879 i
\end{array}\right. \\
& -0.0004-0.1425 i-0.0111+0.0963 i-0.0161-0.0788 i-0.0055+0.1134 i \\
& -0.0096-0.1043 i \quad 0.0049+0.0833 i]^{\mathrm{T}}
\end{aligned}
$$

\section{Results}

In this paper the matric used against the performance of wireless link is SER or
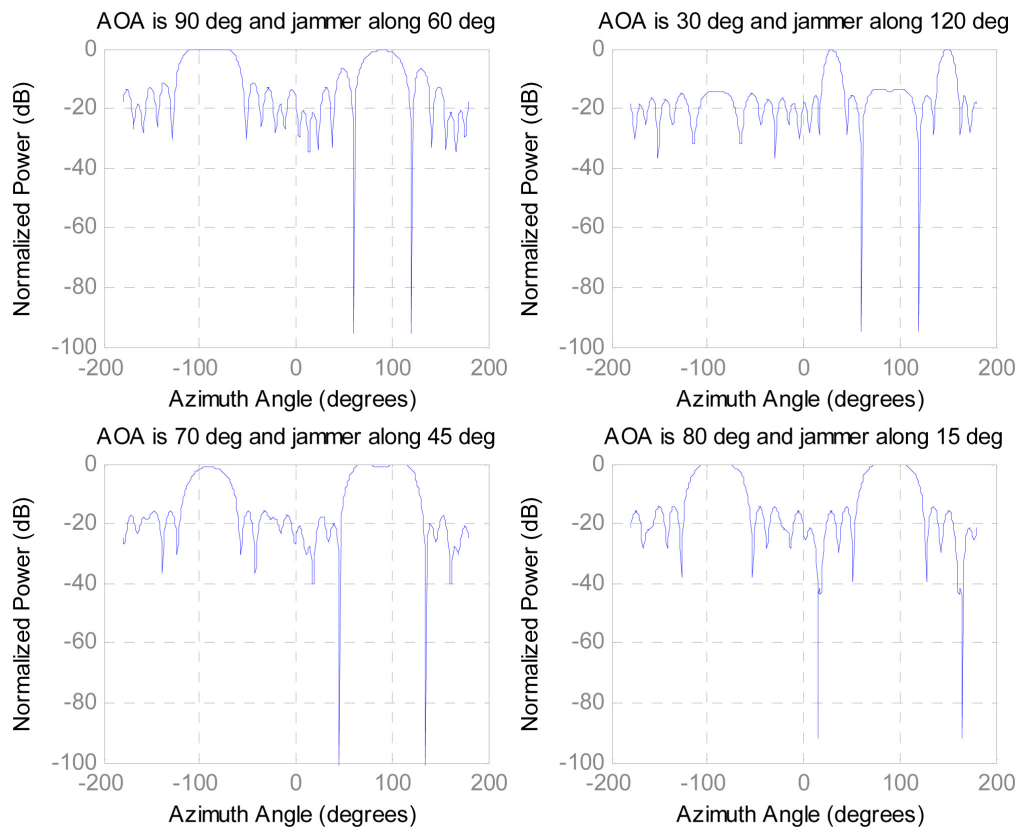

Figure 7. The response of LCMV beamformer under linear array of 10 antenna elements. 
BER for single and 2-hop cases. Figure 8 shows the profile of SER against average SNR for both 2-hop link and single hop link of same path length incorporating AAS of two antenna elements. Here our proposal becomes successful when overall gain (enhancement of desired signal and attenuation of interferences) $G$ is greater than or equal to $3.44 \mathrm{~dB}$ for Nakagami- $m$ fading with $m=4$. In Figure 8 we found that SER of single hop is better than the case of 2-hop for $G=4.77$ and $6.02 \mathrm{~dB}$ but worse than it for $G=3.42 \mathrm{~dB}$. In case of Rayleigh fading the threshold gain $G$ becomes 1.86 visualized from Figure 9 where the SER is found better than 2-hop for $G=1.76,4.77$ and $6.02 \mathrm{~dB}$ but worse than it for $G=$ $1.76 \mathrm{~dB}$.

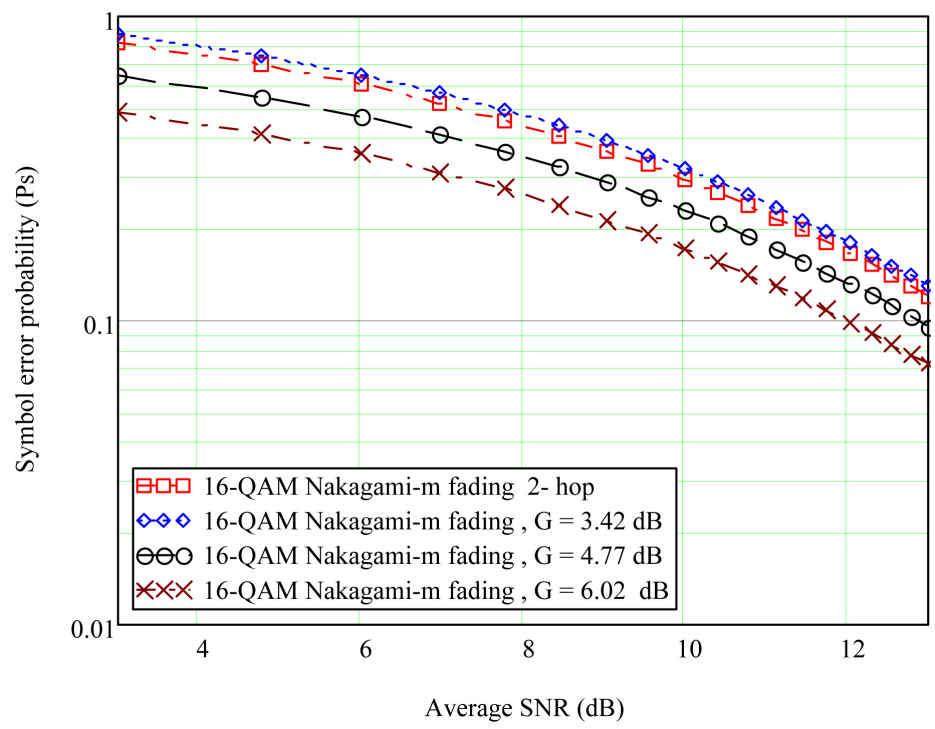

Figure 8. The comparison of SER under 2-hop link and single link with AAS for Nakagami- $m$ fading.

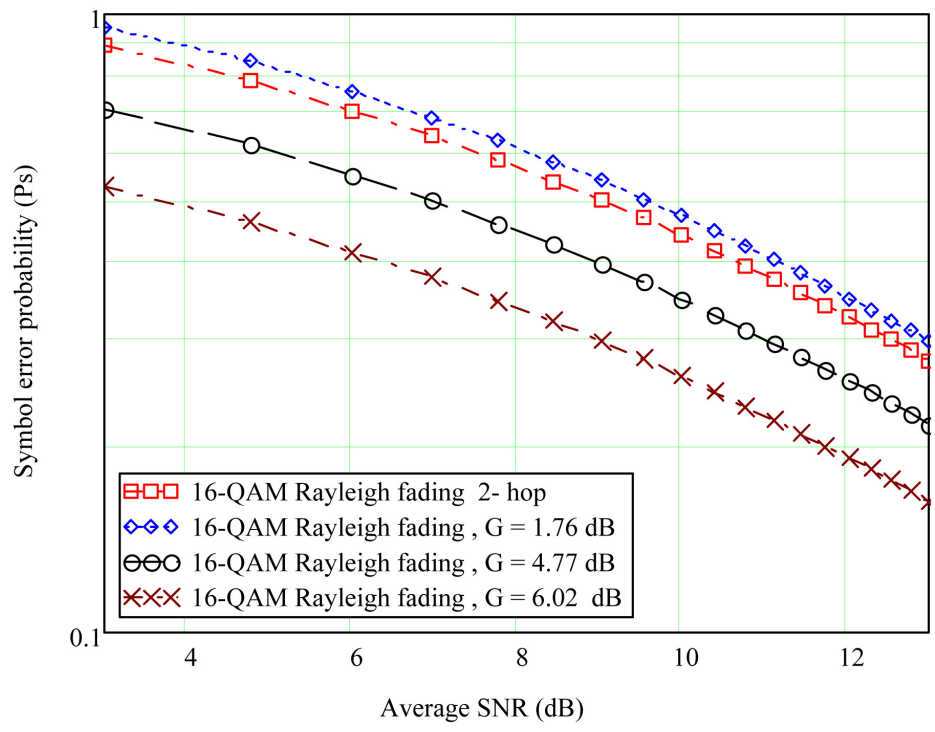

Figure 9. The comparison of SER under 2-hop link and single link with AAS for Rayleigh fading case. 
Finally we compare the theoretical and simulation results regarding 2-hop and single hop AAS under QPSK modulation scheme. We use Rayleigh and Nakagami- $m$ fading condition with bit rate of $9.6 \mathrm{Kbps}$ and Doppler shift of $100 \mathrm{~Hz}$. First the simulation is done for 2000 random symbols shown in Figure 10(a) then for 200,000 symbols shown in Figure 10(b). The simulation curves become smothering for more symbols validates the concept Monte Carlo simulation. The similar result is shown in logarithmic scale in Figure $10(c)$. The difference between simulation and theoretical result is less than 5\% hence ensure $95 \%$ confidence level. All the curves reveal the success of our proposed scheme when we use $G=1.86$ under the simulation as well. Similar job is done for Nakagami- $m$ fading case shown in Figures 11(a)-(c).

The relative performance between Rayleigh and Nakagami- $m$ is found subtle for SNR in the range of $0 \mathrm{~dB}$ to $20 \mathrm{~dB}$. To observe the variation we plot BER

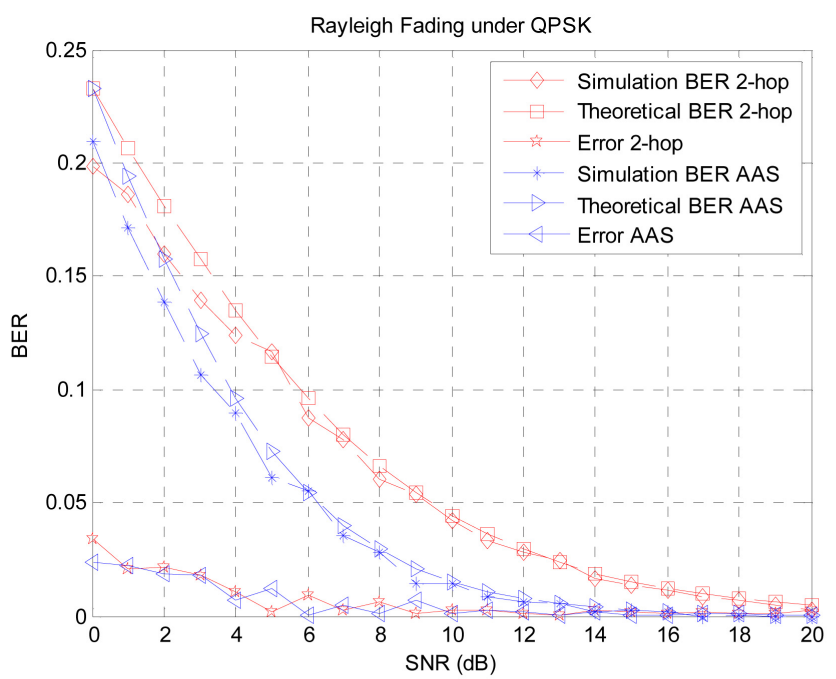

(a)

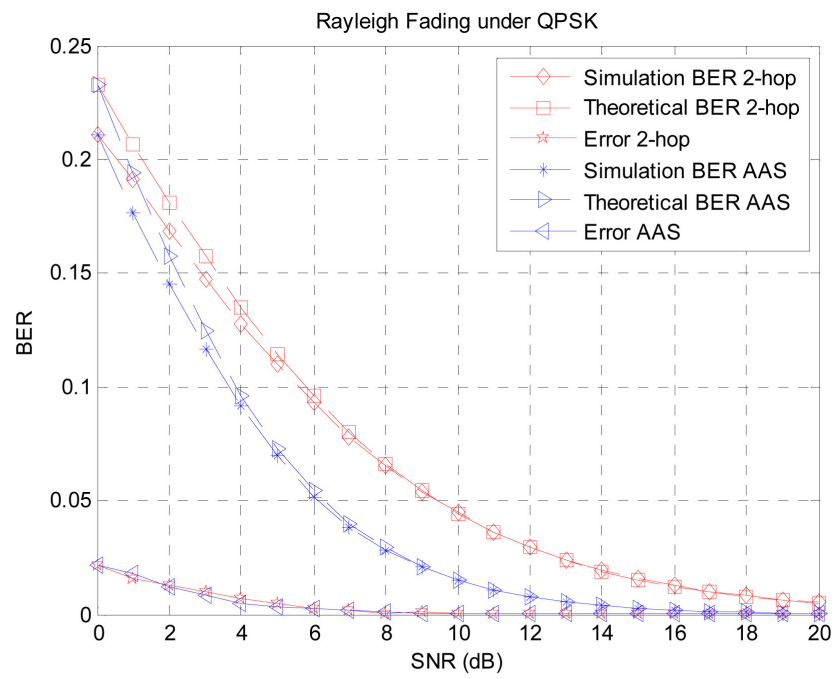

(b)

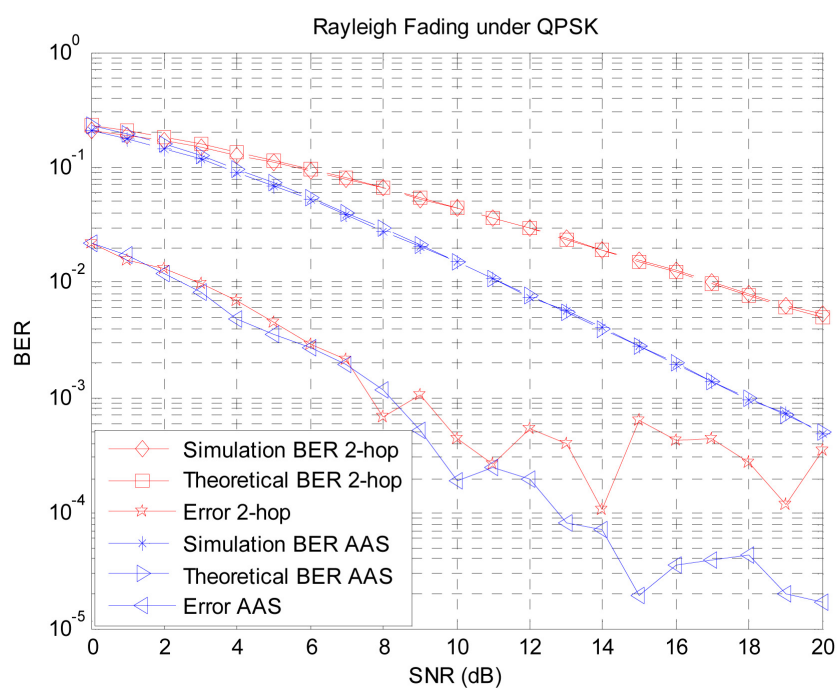

(c)

Figure 10. Comparison of 2-hop and AAS with simulation for Rayleigh fading case. 


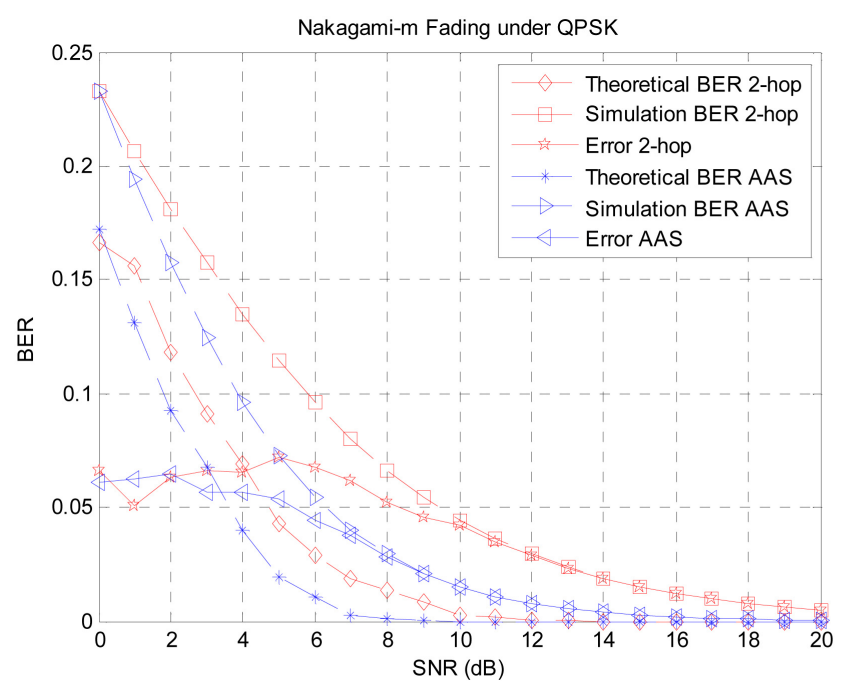

(a)

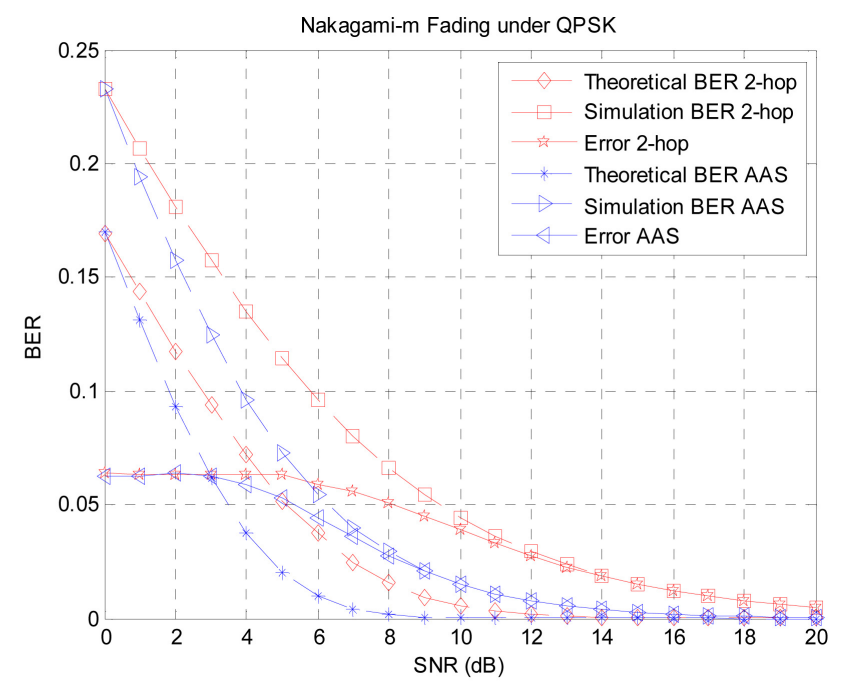

(b)

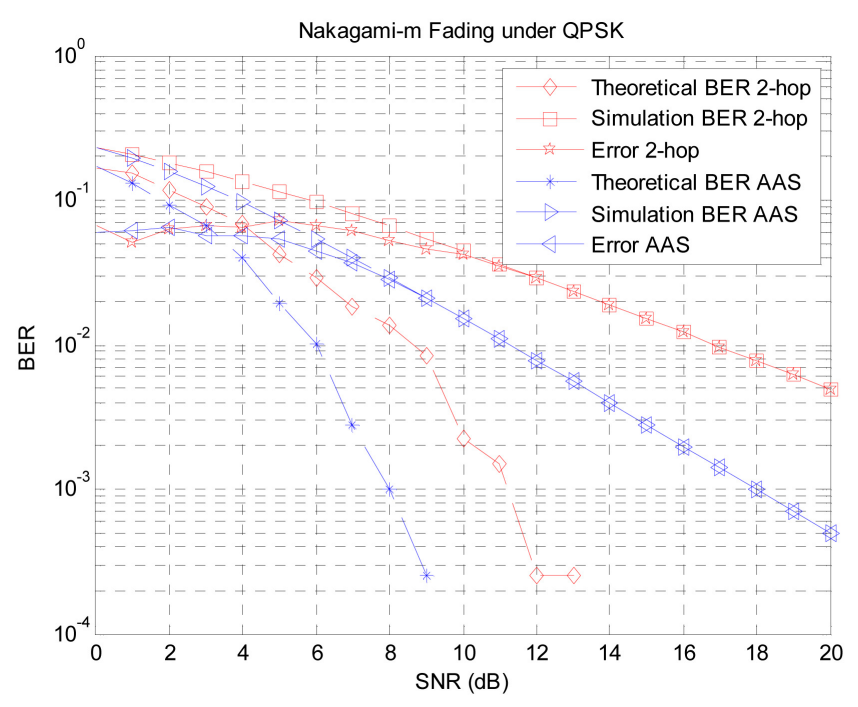

(c)

Figure 11. Comparison of 2-hop and AAS with simulation for Nakagami-m fading case.

against $\mathrm{SNR}$ in the range of $-10 \mathrm{~dB}$ to $0 \mathrm{~dB}$ shown in Figure $12(\mathrm{a})$ and Figure 12(b); where the performance under Nakagami- $m$ fading is found better than that of Rayleigh case because of strong link between transmitter and receiver for former case. Finally the performance of AAS is found better than conventional 2-hop wireless link for SNR in the range of $0 \mathrm{~dB}$ to $20 \mathrm{~dB}$ visualized from Figure 10 and Figure 11. Situation is found reverse for SNR in the range of $-10 \mathrm{~dB}$ to 0 $\mathrm{dB}$ shown in Figure 12(a) and Figure 12(b) taking the same overall array gain. To overcome the above cumbersome situation the array gain has to be changed at low SNR condition even we have to consider the environment of fading.

\section{Conclusion}

The weak radiation of the antenna is strengthened and made more directional using antenna array. The performance of single hop link is compared with 2-hop 


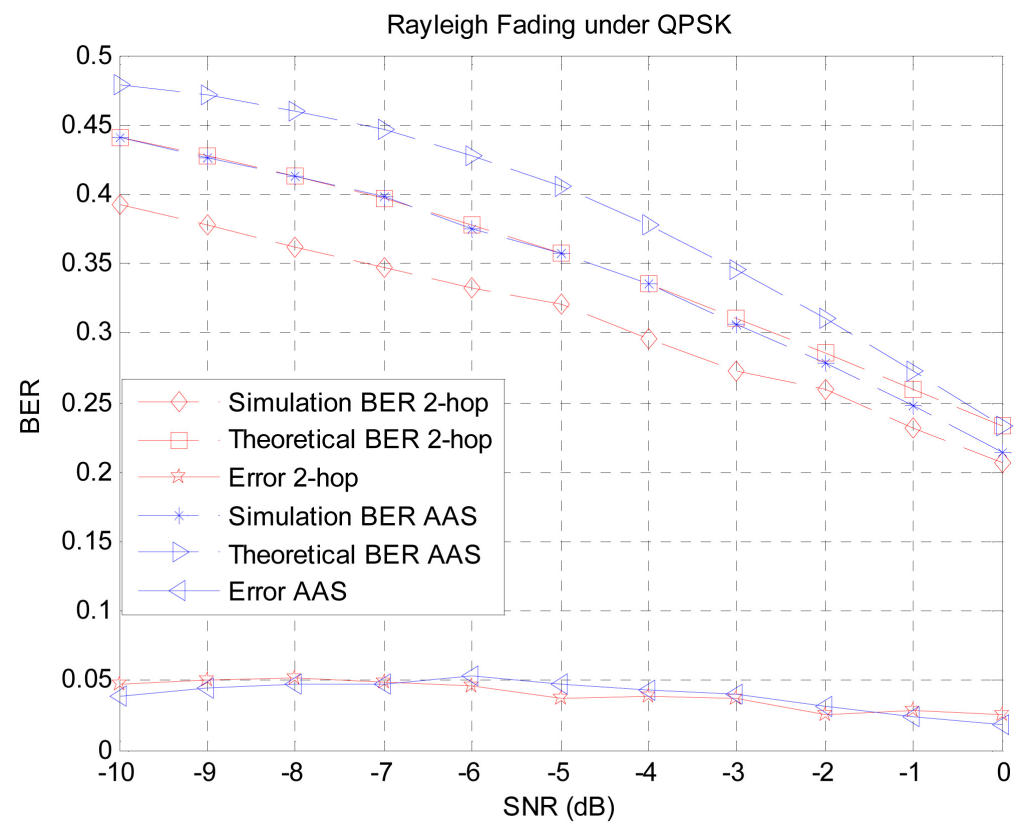

(a)

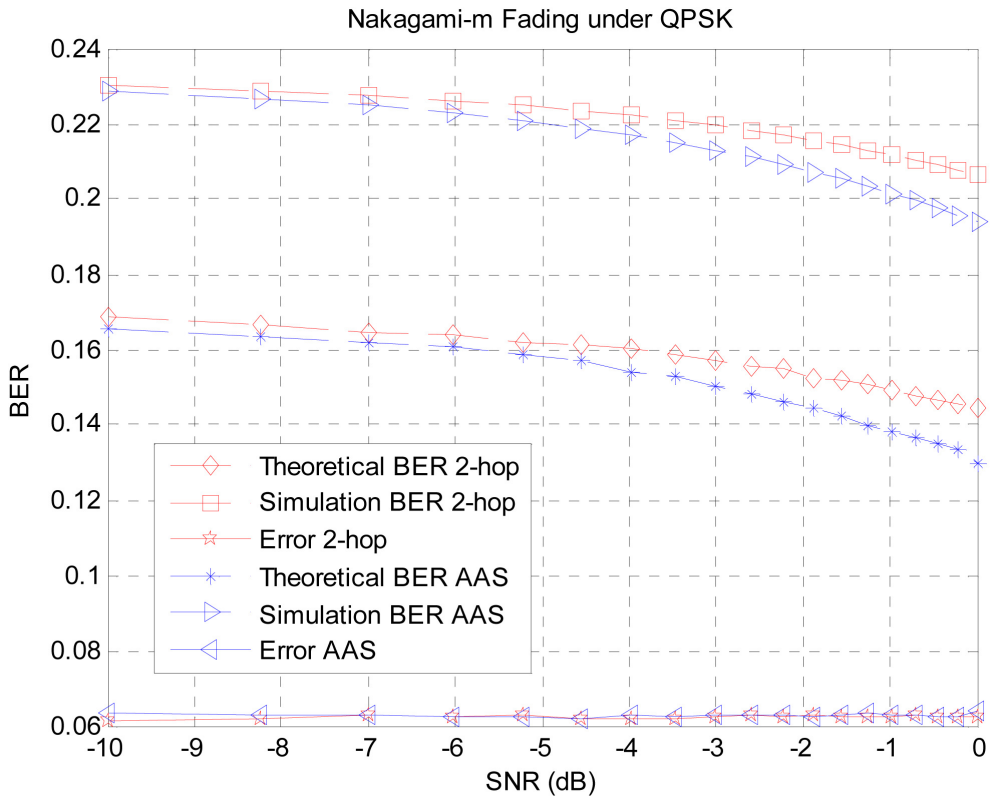

(b)

Figure 12. Comparison of 2-hop link and AAS at low SNR.

link of same distance using adaptive array antenna system. The results of the paper highlighted the threshold gain of the array required to meet the condition of 2-hop wireless link. In this paper we use exponential path loss model suitable for suburban area or a link of short distance applicable in ad-hoc network or femto cell of LTE. Still we have the scope to enhance the model for conventional path loss model of fading channel. The analysis of the paper will be helpful for a wireless network planner to choose the optimum antenna for coverage of WLAN and WMAN. 


\section{Conflicts of Interest}

The authors declare no conflicts of interest regarding the publication of this paper.

\section{References}

[1] Mohammadreza, B. and Umit A. (2017) BER Analysis of Dual-Hop Relaying System with Energy Harvesting in Nakagami-m Fading Channel. 2017 Advances in Wireless and Optical Communications, Riga, Latvia, 96-101.

[2] Bin, L. and Yue, R. (2017) Joint Source and Relay Design for Wireless Powered AF MIMO Relay Systems with Direct Link. 2017 IEEE Globecom Workshops (GC Wkshps), Singapore, 1-6.

[3] Fawaz, S., Caijun, Z., Khalid, A., Hussein, A. and Tharm, R. (2011) Performance Analysis of Fixed-Gain AF Dual-Hop Relaying Systems over Nakagami- $m$ Fading Channels in the Presence of Interference. EURASIP Journal on Wireless Communications and Networking, 2011, 1-10.

[4] Afsana, N., Chowdhuray, A., Imdadul, I. and Amin, M. (2013) Performance Evaluation of Two-Hop Wireless Link under Nakagami-m Fading. International Journal of Advanced Computer Science and Applications (IJACSA), 4, 142-146.

[5] Shubha, S., Madhukumar, A. and Swaminathan, R. (2018) Switching-Based Hybrid FSO/RF Transmission for DF Relaying System. 2018 IEEE Wireless Communications and Networking Conference (WCNC), Barcelona, 15-18 April 2018, 1-6.

[6] Huang, S., Chen, H. and Zhang, Y. (2012) Optimal Power Allocation for Spectrum Sensing and Data Transmission in Cognitive Relay Networks. IEEE Wireless Communications Letters, 1, 26-29. https://doi.org/10.1109/WCL.2012.120211.110056

[7] Huogen, Y., Tang, W. and Li, S. (2013) Outage Probability and SER of Amplify-and-Forward Cognitive Relay Networks. IEEE Wireless Communications Letters, 2, 219-222. https://doi.org/10.1109/WCL.2013.012513.120834

[8] Nuwan, F. and Rajatheva, N. (2013) Effects of Line of Sight Interference on Performance of Amply-and-Forward Relay Network. IEEE Communications Letters, 17, 940-943. https://doi.org/10.1109/LCOMM.2013.040913.130328

[9] Krikidis, I., Thompson, J., Mclaughlin, S. and Goertz, N. (2009) Max-Min Relay Selection for Legacy Amply-and-Forward Systems with Interference. IEEE Transactions on Wireless Communications, 8, 3016-3027. https://doi.org/10.1109/TWC.2009.080383

[10] Suraweera, H., Garg, H. and Nallanathan, A. (2010) Performance Analysis of Two Hop Amplify-and-Forward Systems with Interference at the Relay. IEEE Communications Letters, 14, 692-694. https://doi.org/10.1109/LCOMM.2010.08.100109

[11] Ahasanun, N., Qinghai, Y. and Kyung-sup, K. (2011) Performance Analysis of Two-Hop Cooperative MIMO Transmission with Best Relay Selection in Rayleigh Fading Channel. The International Arab Journal of Information Technology, 8, 9-15.

[12] Zhuo, C., Jinhong, Y. and Branka, V. (2005) Analysis of Transmit Antenna Selection/Maximal-Ratio Combining in Rayleigh Fading Channels. IEEE Transactions on Vehicular Technology, 54, 1312-1321. https://doi.org/10.1109/TVT.2005.851319

[13] Nan, Y., Phee, L., Maged, E., Robert, S. and Iain, B. (2013) Transmit Antenna Selection for Security Enhancement in MIMO Wiretap Channels. IEEE Transactions on Communications, 61, 144-154. https://doi.org/10.1109/TCOMM.2012.12.110670 
[14] Godara, L. (1997) Application of Antenna Arrays to Mobile Communication, Part I: Performance Improvement, Feasibility and System Considerations. Proceedings of the IEEE, 85, 1033-1060.

[15] Godara, L. (1997) Application of Antenna Arrays to Mobile Communication, Part II: Beamforming and Direction of Arrival Consideration. Proceedings of the IEEE, 85, 1195-1245. https://doi.org/10.1109/5.622504

[16] Rappaport, T. (1991) Wireless Personal Communications: Trends and Challenges. IEEE Antennas and Propagation Magazine, 33, 19-29. https://doi.org/10.1109/74.97946

[17] Freweyni, K., Tawatu, A. and Dilip, M. (2013) Smart Antenna Techniques for Interference Suppression in WCDMA Using LMS Algorithm. International Journal of Computing Science and Communication Technologies, 6, 916-922.

[18] Rana, L., Anum, A., Anis, R., Shahid, A. and Shahzad, A. (2011) Adaptive Beamforming Algorithms for Anti-Jamming. International Journal of Signal Processing, Image Processing and Pattern Recognition, 4, 95-105.

[19] Feng, S. and Jinyang, S. (2015) A Robust Adaptive Beamformer Based on Semidefinite Programming with Quadratic Constraints. International Journal of Hybrid Information Technology, 8, 345-354. https://doi.org/10.14257/ijhit.2015.8.2.32

[20] Somnath, P., Abhisek, P., Nisha, N., Sujeet, K., Vikky, J. and Monoj, K. (2015) Power Pattern Synthesis of Smart Antenna Array Using Different Adaptive Algorithms. International Journal of Advanced Research, 3, 1459-1466.

[21] Emmanuel, C. and Barrie, W. (2004) Digital Signal Processing: A Practical Approach. 2nd Edition, Pearson Education, London.

[22] Widrow, B. and Samuel, D. (2005) Adaptive Signal Processing. Pearson Education, London.

[23] Haque, S., Ariful, M., Imdadul, I. and Amin, M. (2007) Radiation Pattern and Beamwidth Control of Linear and Rectangular Array Antenna System. Proceedings of the East West University Journal, 1, 119-128.

[24] Jahan, J., Imdadul, I. and Mostafizur, R. (2015) Adaptive Beamforming and Cancellation of Jammer Using Linear Array Antenna System. International Journal of Engineering Sciences \& Research Technology, 4, 648-657.

[25] Chiu, C., Chen, C.-H., Liao, S.-H. and Chen, K.-C. (2012) Bit Error Rate Reduction by Smart UWB Antenna Array in Indoor Wireless Communication. Journal of Applied Science and Engineering, 15, 139-148.

[26] Rim, H. (2013) BER Performance of Antenna Array-Based Receiver Using Multi-User Detection in a Multipath Channel. International Journal of Wireless \& Mobile Networks, 5, 94-102.

[27] Feng, S., Fengfeng, C. and Jinyang, S. (2015) Robust Adaptive Beamforming Based on Steering Vector Estimation and Covariance Matrix Reconstruction. Communications Letters, 19, 1636-1639.

[28] Simon, H. (2013) Adaptive Filter Theory. 5th Edition, Prentice Hall, Upper Saddle River.

[29] Xiaojun, M., Wenxing, L., Yingsong, L., Yaxiu, S. and Zhuqun, Z. (2015) Robust Adaptive Beamforming against Signal Steering Vector Mismatch and Jammer Motion. International Journal of Antennas and Propagation, 2015, Article ID: 780296.

[30] Ahmed, M., Osama, M., Ghandour, F. and Hesham, F. (2015) Performance Enhancement for Adaptive Beam-Forming Application Based Hybrid PSOGSA Algorithm. Journal of Electromagnetic Analysis and Applications, 7, 126-133. https://doi.org/10.4236/jemaa.2015.74014 